\title{
Crescimento de crianças nascidas prematuras
}

\author{
Growth of preterm-born children
}

Adriane de Andre Cardoso-Demartini', Antonio Carlos Bagatin', Regina Paula Guimarães Vieira Cavalcante da Silva', Margaret Cristina da Silva Boguszewski'

1 Departamento de Pediatria, Hospital de Clínicas, Universidade Federal do Paraná (UFPR), Curitiba, PR, Brasil
Correspondência para: Margaret Cristina da Silva Boguszewski

Hospital de Clínicas,

Departamento de Pediatria

Rua General Carneiro, 181

80060-150 - Curitiba, PR, Brasil

margabogus@uol.com.br

Recebido em 16/Set/2011

Aceito em 21/Out/2011

\section{SUMÁRIO}

Crianças nascidas prematuras podem passar por um período de restrição do crescimento logo após o nascimento. A normalização do crescimento tem início nos primeiros meses de vida, podendo ocorrer de forma lenta e progressiva. Muitas vezes essas crianças mantêm-se mais baixas e com menor peso durante toda a infância quando comparadas àquelas nascidas a termo. Em alguns casos, a recuperação completa só ocorre na adolescência. Entretanto, algumas crianças não conseguem recuperar totalmente o ganho de peso e altura, e adultos nascidos prematuros apresentam maior risco de baixa estatura. 0 comprometimento do crescimento é mais significativo naquelas nascidas prematuras e pequenas para a idade gestacional. Fatores como estatura-alvo, peso ao nascimento, idade gestacional, intercorrências neonatais e escolaridade materna interferem no potencial de crescimento. Especial atenção deve ser dada aos nascidos prematuros durante todo o período de crescimento. Arq Bras Endocrinol Metab. 2011;55(8):534-40

\section{Descritores}

Crescimento; recém-nascido prematuro; prematuridade; baixo peso ao nascimento; pequeno para a idade gestacional; recuperação do crescimento; curvas de crescimento

\section{SUMMARY}

Children born prematurely might experience a period of growth restriction just after birth. Catch-up growth begins during the first months of life and can be slow and progressive. These children may remain shorter and thinner throughout infancy and childhood compared to children born at term. In some cases, complete catch-up growth occurs only during adolescence. However, some children do not completely recover growth, and adults born prematurely are at increased risk of short stature. Impaired growth is more frequent in those born preterm and small for gestational age. Factors such as target height, birth weight, gestational age, neonatal morbidities and maternal education interfere in growth potential. Special attention should be given to children born preterm during the whole growth period. Arq Bras Endocrinol Metab. 2011;55(8):534-40

\section{Keywords}

Growth; preterm newborn; premature children; low birthweight; prematurity; small for gestational age; catch-up growth

\section{INTRODUÇÃO}

A tualmente, cerca de 13 milhões de crianças nascem prematuramente em todo o mundo e o número de partos prematuros tem aumentado nos últimos anos (1). A melhoria da assistência obstétrica e o aumento do número de gestações múltiplas, provavelmente em decorrência das técnicas de reprodução assistida, são alguns fatores que contribuem para esse aumento. Dos óbitos que ocorrem no período neonatal, não relacio- nados a malformações congênitas, $28 \%$ resultam de nascimentos prematuros. Estima-se um gasto de mais de 26 bilhões de dólares apenas nos Estados Unidos com problemas relacionados à prematuridade $(1)$.

A etiologia da prematuridade é multifatorial e varia de acordo com a idade gestacional (IG). Aproximadamente $14 \%$ dos casos podem ser explicados por fatores maternos e $11 \%$ por fatores genéticos fetais (2). Infecções maternas predominam como causa de nascimento 
prematuro entre 24 e 32 semanas de gestação, e o estresse e a distensão abdominal excessiva são predominantes entre 32 e 37 semanas (3). No entanto, os fatores mais frequentemente relacionados ao nascimento prematuro espontâneo é a história prévia de trabalho de parto prematuro e o baixo nível socioeconômico da mãe (4). Entre $40 \%$ e $50 \%$ dos nascimentos prematuros são considerados idiopáticos (1).

Os contínuos avanços em terapia intensiva neonatal levaram à diminuição da mortalidade de recém-nascidos prematuros (RNPTs), com peso de nascimento (PN) e IG progressivamente menores, aumentando a preocupação de neonatologistas, pediatras e familiares com a qualidade da vida dessas crianças, seu crescimento somático e seu desenvolvimento neuropsicomotor.

O crescimento é um processo contínuo, complexo, resultante da interação de fatores genéticos, nutricionais, hormonais e ambientais. Diante dessa nova realidade de sobrevida dos prematuros, é difícil prever como será o crescimento de um RNPT de muito baixo peso. São crianças que podem apresentar muitas intercorrências clínicas, que aumentam o gasto energético e as necessidades nutricionais, e ainda enfrentam sérias restrições na oferta e/ou no aproveitamento dos nutrientes. Nas crianças nascidas prematuras, a deficiência pôndero-estatural observada nos primeiros meses de vida pode persistir por toda a infância, adolescência e idade adulta. Neste artigo, o crescimento de prematuros nessas diferentes fases é revisado, assim como definições importantes para o melhor entendimento das situações a que essas crianças estão sujeitas. As curvas de crescimento específicas para essa população também são mencionadas, uma vez que devem ser utilizadas para um diagnóstico adequado e acompanhamento das crianças nascidas prematuras.

\section{CURVAS DE CRESCIMENTO PARA PREMATUROS}

As curvas de crescimento são recursos utilizados para avaliar o crescimento e o estado nutricional das crianças. O padrão de crescimento representado nas curvas indica o crescimento ideal para uma população saudável e pode ter caráter prescritivo. As curvas utilizadas para a avaliação antropométrica de RNs de diferentes idades gestacionais são construídas a partir de informações obtidas de crianças nascidas com diferentes idades gestacionais (curvas de crescimento intrauterino) ou a partir de medidas fetais obtidas por meio de ultrassonografia, também em diferentes idades gestacionais (curvas de crescimento fetal). Com os avanços no atendimento de prematuros nas últimas décadas, o padrão de crescimento de crianças nascidas prematuras também mudou. Por essa razão, as curvas construídas com medidas de crianças nascidas após 1990 são as mais adequadas.

As curvas de crescimento intrauterino são as mais recomendadas para o acompanhamento de crianças prematuras e existem pelo menos 25 curvas desse tipo disponíveis (7). Um exemplo desse tipo de curva é a de Babson e Benda publicada em 1976 (8), que, além do padrão de crescimento intrauterino, inclui também o crescimento de crianças nascidas a termo, por isso denominada pelos autores de "fetal-infant growth graph". É uma curva bastante usada, apesar de apresentar algumas limitações, inclusive o fato de ter sido criada há mais de 30 anos. Na construção da curva foram avaliados apenas 45 RNPTs com IG abaixo de 30 semanas, sendo que, na curva, a IG começa em 26 semanas, impedindo o uso para prematuros abaixo dessa idade. Outro problema é que o aumento do peso acontece em intervalos de $500 \mathrm{~g}$, dificultando a avaliação de mudanças menores do peso. Com o objetivo de atualizar a curva e corrigir as limitações, uma revisão sistemática da literatura foi realizada e as informações obtidas foram incluídas na curva original de Babson e Benda (9). Foram selecionadas informações de três estudos, um canadense, um sueco e um australiano. Além disso, dados da curva de crescimento do Centers for Disease Control (CDC 2000) foram incluídos para complementar a curva após a $40^{a}$ semana de gestação (10). Com a versão atualizada, é possível avaliar RNs a partir de 22 semanas de gestação e até 10 semanas após o termo (9). A desvantagem desse tipo de curva recai no fato de que crianças nascidas prematuras geralmente são menores do que fetos da mesma IG que nascerão a termo (11-13).

As curvas de crescimento fetal são construídas a partir de medidas do feto obtidas por ultrassonografia e, teoricamente, refletem melhor o crescimento esperado para cada IG, já que eliminaria os efeitos da prematuridade sobre o peso do RN. A dificuldade desse tipo de avaliação recai na sensibilidade da ultrassonografia em avaliar o peso fetal, especialmente em idades gestacionais menores (7).

Nos Estados Unidos, uma nova curva já foi criada e validada, sendo gênero-específico devido às diferenças encontradas no tamanho ao nascimento entre meninos e meninas, neste (14) e em outros estudos $(15,16)$. Um estudo internacional multicêntrico foi iniciado recentemente para a construção de uma curva-padrão para 
a avaliação do crescimento pós-natal dos prematuros e deverá estar disponível nos próximos anos (17), na tentativa de superar os problemas encontrados nas curvas existentes até o momento. Esse novo modelo propõe uma curva multiétnica e conceitualmente muito próxima às novas curvas da Organização Mundial de Saúde (OMS) $(13,18)$.

Após completar 10 semanas de idade a partir do termo, as curvas da OMS de 2006 são recomendadas, sendo necessário corrigir a idade cronológica para a prematuridade pelo menos até os 3 anos de idade. A curva da OMS foi construída com dados de crianças saudáveis de diferentes países, inclusive o Brasil, alimentadas com leite materno até os 4 meses de idade (18).

\section{CLASSIFICAÇÃO}

Define-se como prematuro todo nascimento que ocorre com IG inferior a 37 semanas (mais de 196, porém menos de 259 dias completos de gestação) e como prematuro extremo aqueles nascidos com IG menor que 28 semanas (menos de 196 dias completos de gestação) (5).

De acordo com o tamanho ao nascimento, os RNs podem ser classificados como (6): a) adequados para a IG (AIG): peso e/ou comprimento entre +2 DP e -2 DP; b) pequenos para a IG (PIG): peso e/ou comprimento ao nascimento $\leq-2 \mathrm{DP} ; \mathrm{c}$ ) grande para a IG (GIG): peso e/ou comprimento ao nascimento $\geq+2 \mathrm{DP}$.

Assim, RNs prematuros podem ser também AIG, PIG ou GIG, situações que podem interferir na evolução clínica.

A estimativa da IG geralmente é feita por meio da data da última menstruação e da ultrassonografia obstétrica realizada no primeiro trimestre de gestação, além do exame físico do RN.

\section{CRESCIMENTO PRÉ-NATAL}

$\mathrm{Na}$ fase intrauterina, o crescimento fetal é determinado principalmente por fatores genéticos, nutricionais e hormonais, como o IGF-1 (insulin-like growth factor 1), IGF-2, $\operatorname{IGFBP}_{3}$ (IGF binding protein) e insulina (19). Pode ser dividido em três fases $(20,21)$ : a) primeira fase, da concepção ao quarto mês, em que predomina a hiperplasia com aumento do número de células; b) segunda fase, do quarto ao sétimo mês, em que, além da hiperplasia celular, ocorre a hipertrofia, já iniciando um aumento do tamanho das células; c) terceira fase, do sétimo mês ao termo, com predomínio da hipertrofia.
Dessa forma, os RNPTs são privados desse período de intenso crescimento intrauterino em que deveriam estar recebendo todos os nutrientes de forma equilibrada, quando a velocidade de crescimento seria mais de 10 vezes superior à encontrada durante o estirão da puberdade (22). Passar por essa fase fora do ambiente uterino pode resultar em um desenvolvimento mais lento e tardio (23).

\section{CRESCIMENTO NO PERÍODO NEONATAL}

Após o nascimento, a criança precisa regular sua própria temperatura e cessa o fluxo constante de nutrientes e oxigênio através da placenta. $\mathrm{O}$ crescimento extrauterino não pode ser comparado ao crescimento fetal. O ganho de peso é influenciado pela oferta calórica, enquanto o aumento do comprimento e do perímetro cefálico (PC) é determinado pela oferta de proteínas. Os RNPTs possuem um padrão próprio de crescimento e este é classificado em quatro fases, que variam de dias a semanas, em função da IG, do $P N$ e da duração da doença inicial.

A primeira fase é a do retardo do crescimento e corresponde à fase de doença inicial, logo após o nascimento. Ocorre um desvio do gasto energético para manter funções vitais à sobrevida fora do ambiente uterino em detrimento do gasto necessário para manter o crescimento. Ocorre perda de peso, que pode ser comparada à perda de peso inicial do RN a termo (RNT) sadio, mas que apresenta intensidade e duração inversamente relacionada à $\mathrm{IG}$ e ao $\mathrm{PN}$ e diretamente relacionada à gravidade do quadro neonatal $(20,24)$. Ou seja, quanto menor a IG e o PN e quanto mais grave a situação clínica, maior a perda de peso. O peso mais baixo é encontrado entre o $4^{\circ}$ e o $7^{\circ}$ dia de vida $(2,25)$.

A segunda fase, ou fase de transição, ocorre com a estabilização das intercorrências clínicas e com o melhor aporte calórico. O RNPT para de perder peso e há um discreto aumento do PC e do comprimento (20). O PN geralmente é recuperado entre o $8^{\circ}$ e o $24^{\circ}$ dia de vida, sendo a recuperação mais precoce quanto maior o PN (2).

A terceira fase, de recuperação (catch-up), caracteriza-se por um aumento rápido do peso, comprimento e PC, com velocidade de crescimento acelerada, ultrapassando os valores estimados nas curvas de crescimento intrauterino ou pós-natal $(19,20,23)$. Define-se recuperação do crescimento como a variação do escore $\mathrm{Z}$ ou desvio-padrão $(\mathrm{DP}) \geq 0,67$, correspondendo à as- 
censão de um canal na curva de percentis (26) ou como a recuperação acima do -2 DP nas curvas de referência (27). A recuperação ocorre primeiramente no PC, até os 12 meses de vida, seguida pela recuperação do comprimento e, por último, do peso (23). A aceleração do crescimento geralmente é máxima entre 36 e 40 semanas pós-concepção e $80 \%$ dos prematuros atingem a normalidade nas curvas de referência para estatura e PC até os 3 anos de idade e 70\% para o peso, estando a ausência de recuperação associada ao baixo nível socioeconômico e à ocorrência de doenças (28).

A quarta e última fase, de homeorrexe ou de equilíbrio, é caracterizada por uma velocidade de crescimento comparável à das crianças saudáveis nascidas a termo (20). É importante ressaltar que quanto menor a IG e menor o PN, maior será a perda de peso inicial e maior o tempo para recuperá-lo, resultando em aumento da gravidade $(23,29)$.

Alguns fatores estão associados ao maior risco de comprometimento do crescimento do prematuro, entre os quais a displasia broncopulmonar (30-32), enterocolite necrosante (32), uso pós-natal de corticoide (32-34), retardo do crescimento intrauterino (RCIU) ou RN PIG (35-37), sexo masculino $(32,36)$, hipertensão arterial materna (38) e altos níveis de fosfatase alcalina durante o período neonatal $(38,39)$.

\section{CRESCIMENTO NA INFÂNCIA E NA ADOLESCÊNCIA}

Aproximadamente $80 \%$ dos prematuros apresentam recuperação do crescimento nos primeiros 2 anos de vida $(2,23,39)$. Entretanto, a recuperação tardia do crescimento, até os 14 anos de idade $(23,40-42)$, tem sido descrita. Sullivan e cols., em 2008, demonstraram que prematuros saudáveis tinham, aos 8 anos de idade, estatura inferior à dos seus pares nascidos a termo, mas que aos 12 anos de idade apenas aqueles nascidos PIG persistiam mais baixos que os controles (43). A recuperação tardia do crescimento, entre 8 e 14 anos de idade, também é descrita em prematuros nascidos com menos de $1.500 \mathrm{~g}$ e IG menor do que 30 semanas, que, mesmo não tendo baixa estatura e baixo peso (peso e estatura abaixo do -2 DP), ainda são mais baixos e mais leves que os controles nascidos a termo e com PN normal (42).

Os fatores relacionados ao crescimento dessas crianças incluem o PN, a ocorrência de problemas neurológicos e doenças neonatais, o tamanho ao nascimento em relação à IG e a estatura dos pais (42).
O tempo em que a recuperação acontece também varia. Em 2010, Funkquist e cols. (25), avaliando 127 RNPTs e AIGs com PN médio de 2.320 g e IG média de 34,3 semanas, demonstraram que o menor peso ocorreu no $7^{\circ}$ dia de vida e o menor comprimento e menor PC aos 14 dias de vida. A recuperação do peso aconteceu após o $11^{\circ}$ dia de vida. Os fatores determinantes para a perda de peso foram o PN e o tempo de nutrição parenteral total (NPT). Já o ganho em altura estava associado ao comprimento ao nascimento e IG, enquanto o PC relacionava-se somente com o PC ao nascimento. Já nas crianças nascidas com menor IG (média 25,9 semanas) e menor PN (média $768 \mathrm{~g}$ ), o comprometimento do crescimento foi mais prolongado, sendo que $8 \%$ apresentavam falência do crescimento entre 40 semanas de idade pós-concepcional ou 4 meses de idade corrigida, $28 \%$ entre 4 e 8 meses e $12 \%$ entre 8 e 20 meses. A ocorrência de doença pulmonar crônica e paralisia cerebral foi o fator associado ao pior padrão de crescimento, não havendo neste grupo, ao contrário do descrito por outros autores, relação com fatores socioeconômicos, PN, IG e tempo de NPT (44). Diferentes épocas de recuperação de peso e comprimento têm sido descritas, inicialmente com melhora do escore $\mathrm{Z}$ do comprimento, porém ainda menor em relação aos controles nascidos a termo, em idades acima dos 12 meses de idade corrigida (45). Em prematuros extremos nascidos com IG média de 25,4 semanas e PN médio de 424 g, a maioria persiste com peso e PC abaixo do $3^{\circ}$ percentil aos 5 anos de idade, enquanto pouco mais da metade já apresenta estatura acima do $3^{\circ}$ percentil. Além do crescimento comprometido, vários apresentam anormalidades no desenvolvimento neuropsicomotor (46). O nascimento prematuro e PIG compromete de forma mais significativa a recuperação do crescimento, e prematuros com menor IG e PIG podem persistir menores que seus pares por muitos anos. Uma recuperação mais rápida do peso, já nos primeiros meses de vida, está associada à maior recuperação do crescimento aos 10 anos de idade (47).

Quanto ao início da puberdade, este pode ser mais precoce nas crianças nascidas prematuramente com baixo peso $(48,49)$. Início em média 10 e 11 meses mais cedo foi descrito nas crianças prematuras nascidas AIGs e PIGs, respectivamente. O pico da velocidade de crescimento também foi mais precoce nos prematuros nascidos PIGs (49). Entretanto, um dos autores sugere que essa antecipação está associada principalmente à 
menor IG (49). Não houve diferença entre a idade da menarca ou mudança de voz entre adolescentes nascidos prematuros e a termo $(49,50)$ e a idade de menarca não teve relação com a estatura adulta (41). A figura 1 ilustra um exemplo em que o estirão da puberdade ocorreu de forma antecipada.

De modo geral, a maioria dos autores sugere que, mesmo havendo recuperação do crescimento, crianças nascidas prematuramente e com PN menor que 1.500 g mantêm-se menores na adolescência se comparadas àquelas nascidos a termo e com PN normal $(42,50)$. A relação direta com a estatura familiar também é observada (23). O retardo de crescimento extrauterino nos primeiros meses de vida, evidenciado pelo fato de alguns RNPTs ainda serem pequenos na $40^{\mathrm{a}}$ semana de idade pós-concepcional, é um fator importante na velocidade de recuperação do crescimento. Crianças nascidas PIGs, com menor IG e com menor PN e aquelas com maior tempo em NPT e com doença respiratória crônica terão maior atraso do crescimento nos primeiros anos de vida, porém pode haver uma recuperação tardia do crescimento.

\section{ESTATURA E COMPOSIÇÃO CORPORAL NA IDADE ADULTA}

Poucos estudos são disponíveis sobre a estatura final de jovens nascidos prematuros. Hack e cols. (36), avaliando 195 jovens aos 20 anos de idade, nascidos com IG média de 29,8 semanas e PN de 1.189 g, demonstraram que apenas para o sexo masculino houve comprometimento do peso e altura, em especial nos nascidos PIGs. Essa diferença não foi encontrada entre as meninas. A menor estatura aos 20 anos estava associada à menor escolaridade materna, à baixa estatura materna e ao menor PN (36). Lundgren e cols., em 2011, também demonstraram essa associação com o nascimento PIG em jovens no momento do alistamento militar, uma vez que $44 \%$ do grupo nascido prematuro e PIG apresentavam altura menor que a do grupo controle, contra apenas $4,4 \%$ dos prematuros nascidos AIGs. A menor estatura estava associada a um menor desempenho intelectual (51). Brandt e cols., avaliando aproximadamente 100 jovens entre 17 e 28 anos nascidos prematuros PIGs e AIGs, demonstraram que $54 \%$ dos nascidos PIG permaneciam com altura abaixo da esperada, enquanto

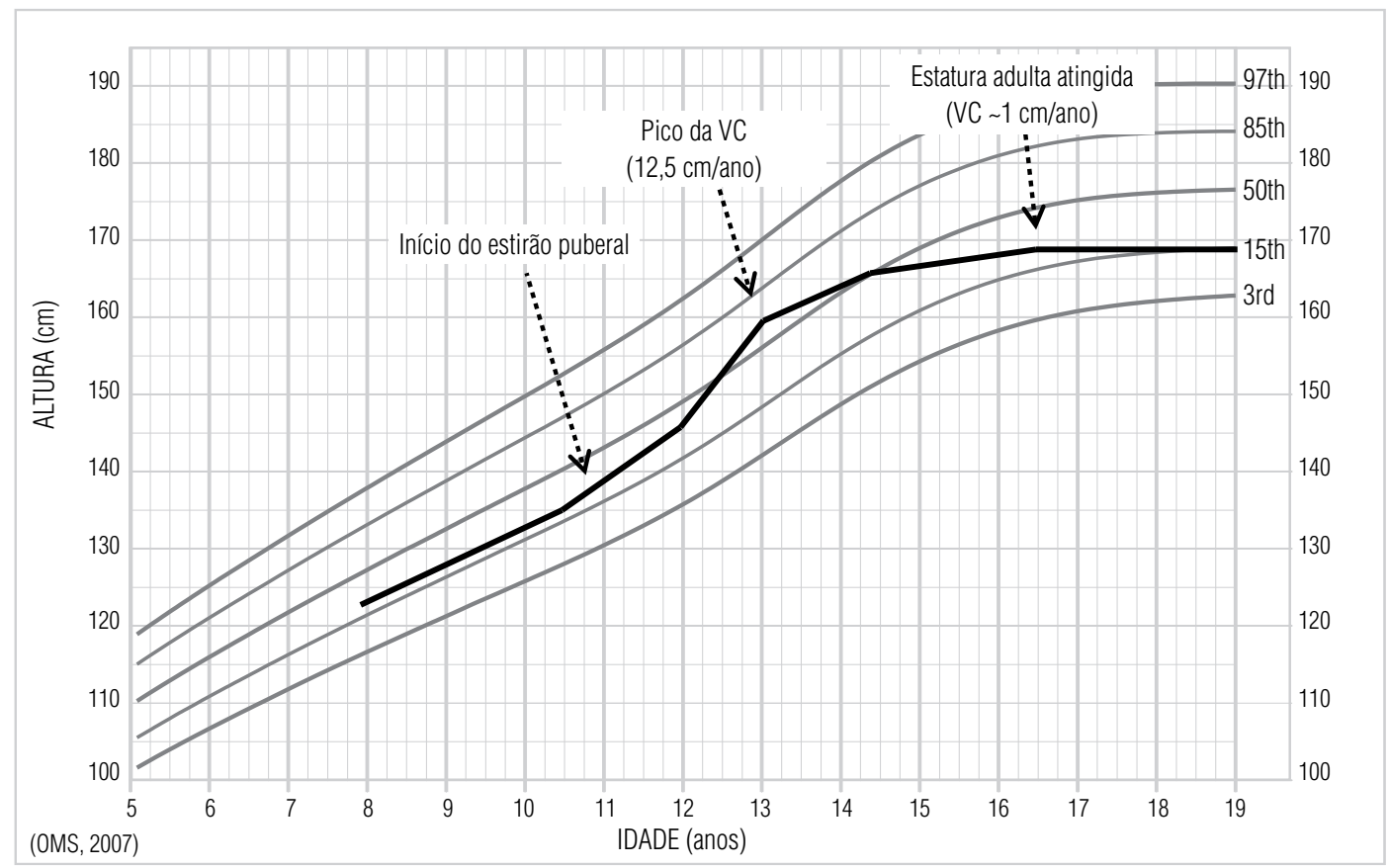

Figura 1. Exemplo de curva de crescimento de paciente do sexo masculino nascido prematuro, mostrando o tempo do estirão da puberdade. A idade no início da aceleração do crescimento foi estimada a partir do momento em que a velocidade de crescimento (VC) aumenta após um período de desaceleração pré-puberal, resultando em aumento superior a 0,3 DP na estatura. 0 pico da VC durante 0 estirão puberal é o ponto em que há 0 maior aumento em estatura, ocorrendo durante 1 ano. A estatura adulta é atingida quando a VC permanece abaixo de $2 \mathrm{~cm} /$ ano, após o término do estirão puberal [Adaptada da ref. 49]. 
todos os nascidos AIGs tinham atingido estatura acima do alvo genético (41).

Entre prematuros extremos, com PN médio de 877 g e IG média de 27,4 semanas, a estatura aos 20 anos de idade foi $3,5 \mathrm{~cm}$ menor para os homens e $3 \mathrm{~cm}$ menor para as mulheres em relação à população geral. Mesmo assim, a estatura aos 20 anos estava adequada à estatura-alvo e o peso estava acima do esperado para a altura, indicando sobrepeso (52).

Mais recentemente, Finken e cols., avaliando 380 prematuros, demonstraram a importância do padrão de crescimento pós-natal sobre a altura adulta. Prematuros nascidos AIGs e que não sofreram restrição do crescimento no período neonatal (restrição extrauterina do crescimento) apresentavam estatura dentro do esperado para a população aos 19 anos de idade. Já os nascidos AIGs e com restrição extrauterina do crescimento apresentavam baixa estatura na vida adulta, semelhante aos nascidos PIGs. A persistência de baixa estatura aos 5 anos de idade foi associada a um risco próximo a $90 \%$ de baixa estatura na idade adulta tanto nos nascidos PIGs como nos AIGs que sofreram restrição do crescimento nos primeiros meses de vida (39).

Com relação ao peso na vida adulta, o ganho parece ser mais pronunciado do que a recuperação da altura, especialmente em mulheres nascidas prematuras $(36,40)$. A combinação de baixo peso ao nascimento seguido por rápida recuperação está associada a doenças cardiovasculares, hipertensão arterial, alterações no metabolismo da glicose, resistência à insulina e diabetes mellitus na idade adulta e deve ser evitada (19,26,53-56).

\section{CONCLUSÃO}

Crianças nascidas prematuras frequentemente apresentam uma falência importante do crescimento no período pós-natal precoce, seguida por recuperação após estabilização clínica. Entretanto, algumas crianças não recuperam o crescimento e podem persistir menores que seus pares nascidos a termo. $\mathrm{O}$ nascimento prematuro e PIGs comprometem de forma mais significativa a recuperação do crescimento. Em alguns jovens nascidos prematuros, a puberdade pode ocorrer um pouco mais cedo que o habitual, sendo este mais um fator que compromete a altura adulta. Curvas de crescimento específicas devem ser utilizadas, e o uso da idade corrigida é recomendado. O nascimento prematuro justifica o acompanhamento clínico regular durante toda a fase de crescimento e desenvolvimento.
Declaração: os autores declaram não haver conflitos de interesse científico neste estudo.

\section{REFERÊNCIAS}

1. Beck S, Wojdyla D, Say L, Betran AP, Merialdi M, Requejo JH, et al. The worldwide incidence of preterm birth: a systematic review of maternal mortality and morbidity. Bull World Health Organ. 2010;88(1):31-8.

2. Euser AM, de Wit CC, Finken MJ, Rijken M, Wit JM. Growth of preterm born children. Horm Res. 2008;70(6):319-28.

3. Gravett MG, Rubens CE, Nunes TM. Global report on preterm birth and stillbirth (2 of 7): discovery science. BMC Pregnancy Childbirth. 2010;10(Suppl 1):S2.

4. Tucker J, McGuire W. Epidemiology of preterm birth. BMJ. 2004;329(7467):675-8.

5. World Health Organization (WHO). International Classification of Diseases (ICD) 10. Avaiable at: http://apps.who.int/classifications/ apps/icd/icd10online/. Accessed: Aug 29, 2009.

6. Battaglia FC, Lubchenco LO. A practical classification of newborn infants by weight and gestational age. J Pediatr. 1967;71(2):159-63.

7. Rao SC, Tompkins J. Growth curves for preterm infants. Early Hum Dev. 2007;83(10):643-51.

8. Babson SG, Benda GI. Growth graphs for the clinical assessment of infants of varying gestational age. J Pediatr. 1976;89(5):814-20.

9. Fenton TR. A new growth chart for preterm babies: Babson and Benda's chart updated with recent data and a new format. BMC Pediatr. 2003;3:13.

10. Kuczmarski RJ, Ogden CL, Grummer-Strawn LM, Flegal KM, Guo SS, Wei R, et al. CDC growth charts: United States. Adv Data. 2000(314):1-27.

11. Bertino E, Milani S, Fabris C, De Curtis M. Neonatal anthropometric charts: what they are, what they are not. Arch Dis Child Fetal Neonatal Ed. 2007;92(1):F7-10.

12. Doubilet PM, Benson CB, Wilkins-Haug L, Ringer S. Fetuses subsequently born premature are smaller than gestational age-matched fetuses not born premature. J Ultrasound Med. 2003;22(4):359-63.

13. Villar J, Knight HE, de Onis M, Bertino E, Gilli G, Papageorghiou AT, et al. Conceptual issues related to the construction of prescriptive standards for the evaluation of postnatal growth of preterm infants. Arch Dis Child. 2010;95(12):1034-8.

14. Olsen IE, Groveman SA, Lawson ML, Clark RH, Zemel BS. New intrauterine growth curves based on United States data. Pediatrics. 2010;125(2):e214-24.

15. Thomas P, Peabody J, Turnier V, Clark RH. A new look at intrauterine growth and the impact of race, altitude, and gender. Pediatrics. 2000;106(2):E21.

16. Niklasson A, Albertsson-Wikland K. Continuous growth reference from 24 th week of gestation to 24 months by gender. BMC Pediatr. 2008;8:8-32.

17. Bertino E, Di Nicola P, Giuliani F, Coscia A, Varalda A, Occhi L, et al. Evaluation of postnatal growth of preterm infants. J Matern Fetal Neonatal Med. 2011;24(Suppl 2):9-11. Epub 2011 Aug 30.

18. WHO Multicentre Growth Reference Study Group. WHO Child Growth Standards based on length/height, weight and age. Acta Paediatr Suppl. 2006;450:76-85.

19. Sauer PJ. Can extrauterine growth approximate intrauterine growth? Should it? Am J Clin Nutr. 2007;85(2):608S-13S.

20. Porto MSA. Crescimento de crianças de risco. In: Comitê de follow-up do RN de alto risco, Sociedade de Pediatria do Estado do Rio de Janeiro, editors. Novo manual de follow-up do recém- 
-nascido de risco. Rio de Janeiro: Serviço de Informação Científica Nestlé; 1995. p. 28-34.

21. Ramos JLA. Crescimento intra-uterino: características e fatores. In: Setian N, editor. Endocrinologia pediátrica: aspectos físicos e metabólicos do recém-nascido ao adolescente. 2.ed. São Paulo: Sarvier; 2002. p. 17-36.

22. Wit JM, Finken MJ, Rijken M, de Zegher F. Preterm growth restraint: a paradigm that unifies intrauterine growth retardation and preterm extrauterine growth retardation and has implications for the small-for-gestational-age indication in growth hormone therapy. Pediatrics. 2006;117(4):e793-5.

23. Rugolo LMSS. Crescimento e desenvolvimento a longo prazo do prematuro extremo. J Pediatr (Rio J). 2005;81(1 Suppl):S101-10.

24. Huysman WA, de Ridder M, de Bruin NC, van Helmond G, Terpstra N, Van Goudoever JB, et al. Growth and body composition in preterm infants with bronchopulmonary dysplasia. Arch Dis Child Fetal Neonatal Ed. 2003;88(1):F46-51.

25. Funkquist EL, TuvemoT, Jonsson B, Serenius F, Nyqvist K. Preterm appropriate for gestational age infants: size at birth explains subsequent growth. Acta Paediatr. 2010;99(12):1828-33.

26. Ong KK, Ahmed ML, Emmett PM, Preece MA, Dunger DB. Association between postnatal catch-up growth and obesity in childhood: prospective cohort study. BMJ. 2000;320(7240):967-71.

27. Lee PA, Chernausek SD, Hokken-Koelega AC, Czernichow P. International Small for Gestational Age Advisory Board consensus development conference statement: management of short children born small for gestational age, April 24-October 1, 2001. Pediatrics. 2003;111(6 Pt 1):1253-61.

28. Monset-Couchard M, de Bethmann O. Catch-up growth in 166 small-for- gestational age premature infants weighing less than 1,000 g at birth. Biol Neonate. 2000;78(3):161-7.

29. Uliani AC, de Carvalho RD, Barros Filho AA. Evolução ponderal de recém-nascidos de muito baixo peso. J Pediatr (Rio J). 1996;72(6):388-93.

30. Vohr BR, Bell EF, OhW. Infants with bronchopulmonary dysplasia. Growth pattern and neurologic and developmental outcome. Am J Dis Child. 1982;136(5):443-7.

31. Wheater M, Rennie JM. Poor prognosis after prolonged ventilation for bronchopulmonary dysplasia. Arch Dis Child Fetal Neonatal Ed. 1994;71(3):F210-1.

32. Clark RH, Thomas $P$, Peabody J. Extrauterine growth restriction remains a serious problem in prematurely born neonates. Pediatrics. 2003;111(5 Pt 1):986-90.

33. Gibson AT, Pearse RG, Wales JK. Growth retardation after dexamethasone administration: assessment by knemometry. Arch Dis Child. 1993;69(5 Spec No):505-9.

34. Wood NS, Costeloe K, Gibson AT, Hennessy EM, Marlow N, Wilkinson AR. The EPICure study: growth and associated proble$\mathrm{ms}$ in children born at 25 weeks of gestational age or less. Arch Dis Child Fetal Neonatal Ed. 2003;88(6):F492-500.

35. Gutbrod T, Wolke D, Soehne B, Ohrt B, Riegel K. Effects of gestation and birth weight on the growth and development of very low birthweight small for gestational age infants: a matched group comparison. Arch Dis Child Fetal Neonatal Ed. 2000;82(3):F208-14.

36. Hack M, Schluchter M, Cartar L, Rahman M, Cuttler L, Borawski E. Growth of very low birth weight infants to age 20 years. Pediatrics. 2003;112(1 Pt 1):e30-8.

37. Monset-Couchard M, de Bethmann O, Relier JP. Long term outcome of small versus appropriate size for gestational age co-twins/ triplets. Arch Dis Child Fetal Neonatal Ed. 2004;89(4):F310-4.
38. Fewtrell MS, Cole TJ, Bishop NJ, Lucas A. Neonatal factors predicting childhood height in preterm infants: evidence for a persisting effect of early metabolic bone disease? J Pediatr. 2000;137(5):668-73.

39. Finken MJ, Dekker FW, de Zegher F, Wit JM. Long-term height gain of prematurely born children with neonatal growth restraint: parallellism with the growth pattern of short children born small for gestational age. Pediatrics. 2006;118(2):640-3.

40. Saigal S, Stoskopf B, Streiner D, Paneth N, Pinelli J, Boyle M. Growth trajectories of extremely low birth weight infants from birth to young adulthood: a longitudinal, population-based study. Pediatr Res. 2006;60(6):751-8.

41. Brandt I, Sticker EJ, Gausche R, Lentze MJ. Catch-up growth of supine length/height of very low birth weight, small for gestational age preterm infants to adulthood. J Pediatr. 2005;147(5):662-8.

42. Ford GW, Doyle LW, Davis NM, Callanan C. Very low birth weight and growth into adolescence. Arch Pediatr Adolesc Med. 2000;154(8):778-84.

43. Sullivan MC, McGrath MM, Hawes K, Lester BM. Growth trajectories of preterm infants: birth to 12 years. J Pediatr Health Care. 2008;22(2):83-93.

44. Sices L, Wilson-Costello D, Minich N, Friedman H, Hack M. Postdischarge growth failure among extremely low birth weight infants: Correlates and consequences. Paediatr Child Health. 2007;12(1):22-8.

45. Westerberg AC, Henriksen C, Ellingvag A, Veierod MB, Juliusson $\mathrm{PB}$, Nakstad $\mathrm{B}$, et al. First year growth among very low birth weight infants. Acta Paediatr. 2010;99(4):556-62.

46. Rieger-Fackeldey E, Blank C, Dinger J, Steinmacher J, Bode H, Schulze A. Growth, neurological and cognitive development in infants with a birthweight $<501 \mathrm{~g}$ at age 5 years. Acta Paediatr. 2010;99(9):1350-5.

47. Knops NB, Sneeuw KC, Brand R, Hille ET, den Ouden AL, Wit JM, et al. Catch-up growth up to ten years of age in children born very preterm or with very low birth weight. BMC Pediatr. 2005;5:26.

48. Persson I, Ahlsson F, Ewald U, TuvemoT, Qingyuan M, von Rosen $D$, et al. Influence of perinatal factors on the onset of puberty in boys and girls: implications for interpretation of link with risk of long term diseases. Am J Epidemiol. 1999;150(7):747-55.

49. Wehkalampi K, Hovi P, Dunkel L, Strang-Karlsson S, Jarvenpaa $A L$, Eriksson JG, et al. Advanced pubertal growth spurt in subjects born preterm: the Helsinki study of very low birth weight adults. J Clin Endocrinol Metab. 2011;96(2):525-33.

50. Peralta-Carcelen M, Jackson DS, Goran MI, Royal SA, Mayo MS, Nelson KG. Growth of adolescents who were born at extremely low birth weight without major disability. J Pediatr. 2000;136(5):633-40.

51. Lundgren EM, Tuvemo T, Gustafsson J. Short adult stature and overweight are associated with poor intellectual performance in subjects born preterm. Horm Res Paediatr. 2011;75(2):138-45.

52. Doyle LW, Faber B, Callanan C, Ford GW, Davis NM. Extremely low birth weight and body size in early adulthood. Arch Dis Child. 2004;89(4):347-50.

53. Barker DJ, Gluckman PD, Godfrey KM, Harding JE, Owens JA, Robinson JS. Fetal nutrition and cardiovascular disease in adult life. Lancet. 1993;341(8850):938-41.

54. Cooke RW. Health, lifestyle, and quality of life for young adults born very preterm. Arch Dis Child. 2004;89(3):201-6.

55. Singhal A, Fewtrell M, Cole TJ, Lucas A. Low nutrient intake and early growth for later insulin resistance in adolescents born preterm. Lancet. 2003;361(9363):1089-97.

56. Doyle LW, Anderson PJ. Adult outcome of extremely preterm infants. Pediatrics. 2010;126(2):342-51. 